\title{
Revisiting of Fertilizer Doses in Finger Millet [Eleusine coracana (L.) Garten.] Through Targeted Yield and Soil Test Crop Response (STCR) Approach
}

\author{
Y. Sandhya Rani* , U. Triveni, T.S.S.K. Patro, M. Divya and N. Anuradha \\ Acharya N. G. Ranga Agricultural University, Agricultural Research Station, \\ Vizianagaram - 535 001, A.P., India \\ *Corresponding author
}

\section{A B S T R A C T}

Field experiments were conducted in finger millet for two consecutive

Keywords

Finger millet, Targeted yield Approach, STCR, Recommended dose of fertilizer.

Article Info

Accepted:

23 June 2017

Available Online:

10 July 2017 kharif seasons viz., 2015 and 2016 at Agricultural Research Station, Vizianagaram, Andhra Pradesh with an objective to reschedule the fertilizer doses of finger millet based on Soil Test Crop Response (STCR) and targeted yield approach. The experiment was laid out in Randomized Complete Block Design (RCBD) with three replications. The experimental results revealed that growth characters, yield contributing characters, grain and straw yields and soil available nutrients recorded the highest in the treatment $200 \% \mathrm{RDN}+100 \% \mathrm{RDP}+100 \% \mathrm{RDK}+25 \% \mathrm{RDZn}+25 \%$ $\mathrm{RDS}+25 \% \mathrm{RDB}$ integrated with $5 \mathrm{t} \mathrm{ha}^{-1} \mathrm{FYM}$ which was on par with the treatment in which fertilizers were applied based on STCR equation for a targeted yield of $40 \mathrm{q} \mathrm{ha}^{-1}$ in soils whose nitrogen levels are low (below 140 $\mathrm{kg} \mathrm{ha}^{-1}$ ), high phosphorus levels and medium potassium levels.

\section{Introduction}

Small millets comprising finger millet, kodo millet, foxtail millet, little millet, barnyard millet and proso millet are crops of antiquity known for their drought resistance, resistance to pests and diseases, short growing season as compared to other major cereals and cultivated all-round the year (Devi et al., 2011). Due to all these advantageous characteristics, millet grains are receiving specific attention in the developing countries like India, China and some countries like Africa continent in terms of utilization as food. Millets also contain major and minor nutrients in remarkable amounts.
Finger millet is an important dry land crop, resilient, ability to withstand adverse weather conditions when grown in a variety of soils including sandy, those with high acidity or alkalinity and those having poor water holding capacity.

Finger millet grown on marginal land provides a valuable resource in times of famine. Its grain tastes good and is nutritionally good and rich as it contains high levels of calcium, iron and manganese. The millet straw is also an important livestock feed, building material and fuel. Finger millet 
contains low glycemic index and has no gluten, which makes it suitable for diabetics and people with digestive problems (Treen Hein, 2005).

Soil fertility deterioration owing to excessive removal of nutrients and their inadequate replenishment through fertilizers and manures is considered one of the major causes behind the undesirable fatigue in production and productivity growth rates of different crops. Due to the changes in soil fertility caused by imbalanced fertilizer use, acidity, alkalinity, salinity and declining in soil organic matter, there is every need to continuously monitor the changes in soil properties and adopt the best management practices for maintenance and enhancement of soil health. For enhancing the soil health and sustaining productivity, fertilizer prescriptions based on STCR's targeted yield approach which take into account nutrient demand of the crops for a targeted yield goal and relative contributions from soil and fertilizer sources under a given set of farming situation, revealed inadequacy of the conventional fertilizer recommendation followed.

Intensive crop rotation and imbalanced fertilizer use have resulted in a wide range of nutrient deficiencies in fields. For intensive cropping systems, the current recommended fertilizer rates need revision upward within balance ratio of vital micronutrients specific to crop to enlarge stagnant yields (Tandon, 1997). By supplying plants with micronutrients, either through soil application, foliar spray or seed treatment improved yield, quality and macronutrient efficiency use was improved upto $50 \%$ (Malakouti, 2008). Fertilizer management plays an important role for obtaining satisfactory yield. In order to increase crop productivity nutrient management may be achieved by the involvement of organic sources, bio-fertilizers and micronutrients (Pingali and Pandey, 2005). Micronutrient deficiency can greatly disturb plant yield, quality and the health of domestic animals and humans (Singh et al., 2002). Full exploitation of the genetic potential requires intensive fertilizer application, but it increases the cost of the products. Also about $50 \%$ of applied $\mathrm{N}$ and $70 \%$ of the applied Potassium to the soil remain unavailable to a crop due to a combination of leaching and voltalization. The effective fertilizer recommendation should consider crop needs and nutrients already available in the soil. Among various methods of fertilizer recommendation such as recommended dose of fertilizers (RDF), soil test based recommendation, critical value approach, etc., the soil test crop response (STCR) approach for target yield is unique in indicating both soil test based fertilizer dose and the level of yield that can be achieved with good agronomic practices (Singh et al., 2005).

Although it is suggested that increased application rates of inorganic fertilizers improve finger millet yield and productivity, it is not a practical option for many poor finger millet farmers, as they cannot afford inorganic fertilizer. Therefore, integrated nutrient management (INM) may be a sustainable option for finger millet farmers. The main objectives of INM are improvements in plant performance and resource use efficiency while minimizing negative environmental impacts ( $\mathrm{Wu}$ and $\mathrm{Ma}$ 2015; Chen et al., 2011).

These can be achieved through use of all possible sources of nutrients to meet crop demand, matching soil nutrient availability with crop demand (spatially and temporarily), and minimizing nitrogen losses ( $\mathrm{Wu}$ and $\mathrm{Ma}$, 2015; Drinkwater and Snapp, 2007). The major advantages of INM are increase in yield, water use efficiency, grain quality, economic returns, and sustainability (Wu and Ma, 2015). 


\section{Materials and Methods}

The field experiment was conducted at Agricultural Research Station, Vizianagaram, Andhra Pradesh during Kharif 2015 and 2016. Composite soil sample was drawn from the experimental site at $0-15 \mathrm{~cm}$ depth prior to laying out of the experiment. The soil samples were analyzed by adopting standard procedures. The soil was sandy loam in texture, neutral in reaction, low in organic carbon and available nitrogen, high in available phosphorus and medium in available potassium. The experiment was laid down in Randomized complete block design with following thirteen treatments replicated thrice.

Soil Test Crop Response (STCR) approach was adopted to conduct the field experiment on optimizing of integrated nutrient supply on soil health, growth and yield of finger millet production.

\section{The treatments taken are}

$\mathrm{T} 1: 100 \% \mathrm{RDF}+\mathrm{RDZN}+\mathrm{RDS}+\mathrm{RDB}$

T2: T1+FYM@ 5 tha $^{-1}$

T3: Soil test based fertilizer Recommendation

T4: T3+FYM@ 5 tha $^{-1}$

T5: Based on STCR equation for $35 \mathrm{q}$ ha-1

T6: T5+FYM@ 5 tha $^{-1}$

T7: Based on STCR equation for $40 \mathrm{q}$ ha-1

T8: T7+FYM@ 5 tha $^{-1}$

T9: $150 \% \mathrm{RDN}+100 \% \mathrm{RDP}+100 \% \mathrm{RDK}+$ $25 \%$ RDZn $+25 \%$ RDS $+25 \%$ RDB (90-4030)

T10:T9+FYM@ $5 \mathrm{tha}^{-1}$
T11: $200 \% \mathrm{RDN}+100 \% \mathrm{RDP}+100 \% \mathrm{RDK}+$ $25 \% \mathrm{RDZn}+25 \% \mathrm{RDS}+25 \% \mathrm{RDB}(120-40-$ 30)

T12:T11+FYM@ $5 \mathrm{tha}^{-1}$

T13: Farmers Practice

The fertilizer adjustment equation developed by AICRP on STCR

$\mathrm{FN}=3.76 \mathrm{~T}-0.28 \mathrm{~S} \mathrm{~N}$

$\mathrm{FP}_{2} \mathrm{O}_{5}=1.83 \mathrm{~T}-0.36 \mathrm{~S} \mathrm{P}_{2} \mathrm{O}_{5}$

$\mathrm{FK}_{2} \mathrm{O}=2.17 \mathrm{~T}-0.24 \mathrm{~S} \mathrm{~K}_{2} \mathrm{O}$

Using the above fertilizer adjustment equations, the quantity of fertilizer nutrients required for achieving $35 \mathrm{q} \mathrm{ha}^{-1}$ grain yield and $15 \%$ higher yield $\left(40 \mathrm{q} \mathrm{ha}^{-1}\right)$ of finger millet was worked out

The Recommended dose of fertilizer (RDF) of finger millet in the North Coastal Zone of Andhra Pradesh is 60-40-30 kg NPK ha- ${ }^{-1}$. Soil test based fertilizer recommendation (30\% high or less) depending on the initial soil test values was taken in treatment $\mathrm{T} 3$ and another treatment $\mathrm{T} 4$ with the same fertilizer dose integrated with organic manure (FYM @ $5 \mathrm{t} \mathrm{ha}^{-1)}$ were taken. The fertilizers doses were applied to the treatment T5 based on STCR equation for achieving a target yield of $35 \mathrm{q}$ $\mathrm{ha}^{-1}$ and for target yield of $40 \mathrm{q} \mathrm{ha}^{-1}$ in the treatment T7. Based on the initial soil test values, if the initial soil available nitrogen was low $\left(<140 \mathrm{~kg} \mathrm{ha}^{-1}\right)$ and soil available phosphorus and potassium were medium to high $150 \%$ RDN + 100\% RDP + 100\% RDK $+25 \% \mathrm{RDZn}+25 \% \mathrm{RDS}+25 \% \mathrm{RDB}$ was taken in the treatment $\mathrm{T} 9$ and $200 \% \mathrm{RDN}+$ $100 \%$ RDP $+100 \%$ RDK + 25\% RDZn + $25 \%$ RDS + 25\% RDB in the treatment T11. The treatments $\mathrm{T} 6, \mathrm{~T} 8, \mathrm{~T} 10$ and $\mathrm{T} 12$ were duplications of the treatments T5, T7, T9 and T11 respectively integrated along with organic manure (FYM @ 5 ha ${ }^{-1}$ ). 
After layout of experimental site, calculated quantities of fertilizers and FYM @ $5 \mathrm{t} \mathrm{ha}^{-1}$ were applied as per the treatment details. Fifty percent of nitrogen through urea and entire quantity of phosphorus through SSP (Single Super Phosphate) and potassium through MOP (Murate of Potash) were applied at the time of transplanting as a basal dose to each plot and remaining fifty percent of nitrogen was applied at 30 days after transplanting as indicated in the treatment details.

\section{Results and Discussion}

The growth contributing characters viz., the plant height, No. of productive tillers and leaf length showed significant influence between the treatment with $100 \%$ RDF (control) and other treatments. Significantly highest plant height of $134.1 \mathrm{~cm}$ was recorded in the treatment $200 \% \mathrm{RDN}+100 \% \mathrm{RDP}+100 \%$ $\mathrm{RDK}+25 \% \mathrm{RDZn}+25 \% \mathrm{RDS}+25 \% \mathrm{RDB}$ integrated with FYM @ 5t ha ${ }^{-1}$ (T12) compared to the treatment with $100 \%$ RDF, fertilizers applied with soil test based fertilizer recommendation and farmers practice(T1, T2, T3, T4 and T13).

The leaf length of $35.5 \mathrm{~cm}$ was found highest in the treatment T12 which was on par with treatments $\mathrm{T} 11$ and $\mathrm{T} 7, \mathrm{~T} 8$ in which the fertilizers were applied based on STCR equation for target yield of $40 \mathrm{q} / \mathrm{ha}$ and integrated with FYM @ 5t ha ${ }^{-1}$ compared to the other treatments (Table 1). Similar results were recorded by Kumara et al., (2007) and Anil Kumar et al., (2003). The improvement in growth parameters due to application of fertilizers on STCR basis and application of both fertilizers and organic manures may be attributed due to the increased supply of nutrients (Duryodhana et al., 2004).

The yield contributing characters viz., No. of productive tillers/plant, ear head length and No. of fingers/ear were significantly influenced between the treatments (Table 2). No. of productive tillers/plant were significantly found highest in the treatment with T12 with $200 \%$ RDN + 100\% RDP + $100 \% \mathrm{RDK}+25 \% \mathrm{RDZn}+25 \% \mathrm{RDS}+25 \%$ RDB integrated with FYM @ 5t ha ${ }^{-1}$ (4.0) compared to all the other treatments and found on par with the treatment T11.The ear head length was found highest in the treatment T11 $(9.02 \mathrm{~cm})$ with $200 \% \mathrm{RDN}+$ $100 \% \mathrm{RDP}+100 \% \mathrm{RDK}+25 \% \mathrm{RDZn}+$ $25 \%$ RDS $+25 \%$ RDB and was found on par with the treatments T12, T5, T6, T7, T8, T9 and T10. The No. of fingers/ear was found highest in the treatment T12 (10.1) with $200 \%$ $\mathrm{RDN}+100 \% \mathrm{RDP}+100 \% \mathrm{RDK}+25 \%$ $\mathrm{RDZn}+25 \% \mathrm{RDS}+25 \% \mathrm{RDB}$ integrated with FYM @ 5t ha ${ }^{-1}$ which is on par with the treatments T11 and T8 and significantly different from all the other treatments.

The straw and grain yields of finger millet varied significantly due to application of nutrients on the basis of different approaches (Table 3). The straw and grain yields were found significantly highest in the treatment T12 with $200 \%$ RDN $+100 \%$ RDP $+100 \%$ $\mathrm{RDK}+25 \% \mathrm{RDZn}+25 \% \mathrm{RDS}+25 \% \mathrm{RDB}$ integrated with FYM @ 5t ha ${ }^{-1}$ (88.5 q/ha and $37.35 \mathrm{q} / \mathrm{ha}$ ) which was on par with the treatments with T11 and T7, T8 in which the fertilizers were applied based on STCR equation for target yield of 40q/ha and in integration with FYM @ 5t/ha).

Similar results were recorded by Sankar et al., (2011) and Apoorva et al., (2010). The increase in the yield due to application of fertilizers based on different approaches and integrated nutrient supply was attributed to the increase in growth attributes as a consequent of improved nutrient supply and efficiency of applied nutrients in the soil.

The physic chemical properties (pH and E.C) and Organic carbon percentage (Table 4), 
showed no significant influence with the application of fertilizers through different approaches and integrated nutrient supply
(Srinivasarao et al., 2012; Hemalatha and Chellamuthu 2011; Hemalatha and Chellamuthu 2013).

Table.1 Effect of fertilizer levels on growth characters of finger millet

\begin{tabular}{|l|c|c|c|c|c|c|c|c|c|}
\hline \multirow{2}{*}{ Treatment } & \multicolumn{3}{|c|}{ Plant height $(\mathbf{c m})$} & \multicolumn{3}{c|}{ Leaf length (cm) } & \multicolumn{3}{c|}{ Leaf Width (cm) } \\
\cline { 2 - 11 } & $\mathbf{2 0 1 5}$ & $\mathbf{2 0 1 6}$ & Mean & $\mathbf{2 0 1 5}$ & $\mathbf{2 0 1 6}$ & Mean & $\mathbf{2 0 1 5}$ & $\mathbf{2 0 1 6}$ & Mean \\
\hline T1 & 100.7 & 122.7 & 111.7 & 22.3 & 33.1 & 27.7 & 0.9 & 0.8 & 0.85 \\
\hline T2 & 103.0 & 131.4 & 117.2 & 24.8 & 33.3 & 29.1 & 0.9 & 1.0 & 0.95 \\
\hline T3 & 105.5 & 133.8 & 119.7 & 25.6 & 35.1 & 30.4 & 0.9 & 0.9 & 0.90 \\
\hline T4 & 105.1 & 134.0 & 119.6 & 26.5 & 36.9 & 31.7 & 1.0 & 1.0 & 1.00 \\
\hline T5 & 111.3 & 134.3 & 122.8 & 28.8 & 35.8 & 32.3 & 0.8 & 1.0 & 0.90 \\
\hline T6 & 112.1 & 135.7 & 123.9 & 28.5 & 36.2 & 32.4 & 1.1 & 0.9 & 1.00 \\
\hline T7 & 119.9 & 138.5 & 129.2 & 29.1 & 36.8 & 32.9 & 1.0 & 1.0 & 1.00 \\
\hline T8 & 119.3 & 139.8 & 129.6 & 31.7 & 37.5 & 34.6 & 0.9 & 0.9 & 0.90 \\
\hline T9 & 112.1 & 133.4 & 122.8 & 28.7 & 34.1 & 31.4 & 0.8 & 1.0 & 0.90 \\
\hline T10 & 115.1 & 135.3 & 125.2 & 29.3 & 34.9 & 32.1 & 1.0 & 1.0 & 1.00 \\
\hline T11 & 120.4 & 138.1 & 129.3 & 30.3 & 36.9 & 33.6 & 0.9 & 0.9 & 0.90 \\
\hline T12 & 124.9 & 143.3 & 134.1 & 33.2 & 37.8 & 35.5 & 1.0 & 0.9 & 0.95 \\
\hline T13 & 96.2 & 130.0 & 113.1 & 25.2 & 34.1 & 29.7 & 0.9 & 0.9 & 0.90 \\
\hline SEm \pm & 7.02 & 3.14 & 4.04 & 1.24 & 1.67 & 1.037 & 0.08 & 0.03 & 0.043 \\
\hline CD $(0.05)$ & 20.47 & 9.18 & 11.78 & 3.63 & NS & 3.03 & NS & NS & NS \\
\hline CV\% & 10.92 & 4.04 & 5.68 & 7.69 & 8.16 & 5.65 & 14.99 & 6.02 & 8.03 \\
\hline
\end{tabular}

Table.2 Effect of fertilizer levels on Yield attributes of finger millet

\begin{tabular}{|l|c|c|c|c|c|c|c|c|c|}
\hline \multirow{2}{*}{ Treatment } & \multicolumn{3}{|l}{$\begin{array}{l}\text { No. of productive } \\
\text { tillers/plant }\end{array}$} & \multicolumn{2}{l}{ ear head length (cm) } & \multicolumn{2}{l|}{ No. of fingers/ear } \\
\cline { 2 - 11 } & $\mathbf{2 0 1 5}$ & $\mathbf{2 0 1 6}$ & Mean & $\mathbf{2 0 1 5}$ & $\mathbf{2 0 1 6}$ & Mean & $\mathbf{2 0 1 5}$ & $\mathbf{2 0 1 6}$ & Mean \\
\hline T1 & 2.6 & 2.8 & 2.7 & 7.4 & 7.60 & 7.50 & 7.8 & 8.1 & 7.95 \\
\hline T2 & 2.8 & 3.1 & 2.95 & 8.0 & 7.87 & 7.94 & 8.1 & 8.2 & 8.15 \\
\hline T3 & 2.9 & 2.7 & 2.8 & 8.2 & 7.33 & 7.77 & 8.4 & 7.8 & 8.1 \\
\hline T4 & 2.8 & 2.9 & 2.85 & 8.4 & 7.47 & 7.94 & 8.3 & 8.2 & 8.25 \\
\hline T5 & 2.9 & 2.9 & 2.9 & 8.5 & 8.00 & 8.25 & 8.6 & 8.3 & 8.45 \\
\hline T6 & 2.7 & 3.0 & 2.85 & 8.3 & 8.13 & 8.22 & 8.5 & 8.5 & 8.5 \\
\hline T7 & 3.5 & 3.3 & 3.4 & 9.1 & 8.33 & 8.72 & 9.2 & 8.8 & 9 \\
\hline T8 & 3.6 & 3.4 & 3.5 & 8.8 & 8.47 & 8.64 & 9.4 & 9.1 & 9.25 \\
\hline T9 & 3.2 & 3.1 & 3.15 & 8.4 & 8.20 & 8.30 & 8.5 & 8.5 & 8.5 \\
\hline T10 & 2.8 & 3.5 & 3.15 & 8.6 & 8.27 & 8.44 & 8.6 & 8.7 & 8.65 \\
\hline T11 & 3.8 & 3.7 & 3.75 & 9.5 & 8.53 & 9.02 & 9.8 & 9.2 & 9.5 \\
\hline T12 & 3.9 & 4.1 & 4 & 9.1 & 8.87 & 8.99 & 10.3 & 9.9 & 10.1 \\
\hline T13 & 2.2 & 2.7 & 2.45 & 7.0 & 7.53 & 7.27 & 7.3 & 7.2 & 7.25 \\
\hline SEm \pm & 0.22 & 0.26 & 0.149 & 0.42 & 0.29 & 0.281 & 0.43 & 0.43 & 0.317 \\
\hline CD $(0.05)$ & 0.64 & 0.78 & 0.435 & 1.23 & 0.87 & 0.819 & 1.24 & 1.27 & 0.925 \\
\hline CV\% & 12.39 & 14.6 & 8.29 & 8.71 & 6.44 & 5.91 & 8.52 & 8.88 & 6.39 \\
\hline
\end{tabular}


Table.3 Effect of fertilizer levels on straw and grain yields (q/ha) of finger millet

\begin{tabular}{|l|c|c|c|c|c|c|c|c|c|}
\hline \multirow{2}{*}{ Treatment } & \multicolumn{3}{|c|}{ Straw yield (q/ha) } & \multicolumn{2}{c|}{ Grain yield (q/ha) } & \multicolumn{3}{c|}{ B:C } \\
\cline { 2 - 11 } & $\mathbf{2 0 1 5}$ & $\mathbf{2 0 1 6}$ & Mean & $\mathbf{2 0 1 5}$ & $\mathbf{2 0 1 6}$ & Mean & $\mathbf{2 0 1 5}$ & $\mathbf{2 0 1 6}$ & Mean \\
\hline T1 & 62.7 & 70.5 & 66.6 & 23.8 & 27.4 & 25.63 & 2.10 & 2.40 & 2.25 \\
\hline T2 & 68 & 73.3 & 70.7 & 29.3 & 31.8 & 30.53 & 2.05 & 2.17 & 2.11 \\
\hline T3 & 61.4 & 74.4 & 67.9 & 29.7 & 27.4 & 28.56 & 1.77 & 2.37 & 2.07 \\
\hline T4 & 64.6 & 77.4 & 71.0 & 30.9 & 28.8 & 29.87 & 2.09 & 1.87 & 1.98 \\
\hline T5 & 70.3 & 78.4 & 74.4 & 31.7 & 32.7 & 32.17 & 3.23 & 3.03 & 3.13 \\
\hline T6 & 72.7 & 79.6 & 76.2 & 33.1 & 34.1 & 33.59 & 2.45 & 2.40 & 2.43 \\
\hline T7 & 81.5 & 85.9 & 83.7 & 34.6 & 35.0 & 34.78 & 3.52 & 3.08 & 3.30 \\
\hline T8 & 85.0 & 88.7 & 86.9 & 35.6 & 37.4 & 36.46 & 2.76 & 2.57 & 2.67 \\
\hline T9 & 72.2 & 75.4 & 73.8 & 30.7 & 32.6 & 31.69 & 2.89 & 2.80 & 2.85 \\
\hline T10 & 74.0 & 83.9 & 78.9 & 32.8 & 33.4 & 33.11 & 2.37 & 2.20 & 2.29 \\
\hline T11 & 89.3 & 81.9 & 85.6 & 34.5 & 36.5 & 35.55 & 3.35 & 3.18 & 3.27 \\
\hline T12 & 91.9 & 85.0 & 88.5 & 36.6 & 38.1 & 37.36 & 2.74 & 2.58 & 2.66 \\
\hline T13 & 53.7 & 68.3 & 61.0 & 22 & 24.5 & 23.29 & 1.70 & 2.03 & 1.87 \\
\hline SEm \pm & 5.69 & 2.85 & 3.105 & 2.01 & 1.54 & 1.198 & & & \\
\hline CD $(0.05)$ & 16.63 & 8.33 & 9.061 & 5.8 & 4.5 & 3.498 & & & \\
\hline CV\% & 13.54 & 6.28 & 7.097 & 10.7 & 8.3 & 6.54 & & & \\
\hline
\end{tabular}

Table.4 Effect of fertilizer levels on Soil Physico Chemical properties and OC\% of finger millet

\begin{tabular}{|l|c|c|c|c|c|c|c|c|c|}
\hline \multirow{2}{*}{ Treatment } & \multicolumn{3}{|c|}{ pH } & \multicolumn{3}{|c|}{ EC } & \multicolumn{3}{c|}{ OC\% } \\
\cline { 2 - 10 } & $\mathbf{2 0 1 5}$ & $\mathbf{2 0 1 6}$ & Mean & $\mathbf{2 0 1 5}$ & $\mathbf{2 0 1 6}$ & Mean & $\mathbf{2 0 1 5}$ & $\mathbf{2 0 1 6}$ & Mean \\
\hline T1 & 7.0 & 6.91 & 6.96 & 0.13 & 0.25 & 0.19 & 0.39 & 0.42 & 0.41 \\
\hline T2 & 6.9 & 7.21 & 7.06 & 0.09 & 0.25 & 0.17 & 0.42 & 0.45 & 0.44 \\
\hline T3 & 7.0 & 7.10 & 7.05 & 0.10 & 0.27 & 0.19 & 0.40 & 0.43 & 0.42 \\
\hline T4 & 6.9 & 7.23 & 7.07 & 0.11 & 0.21 & 0.16 & 0.44 & 0.45 & 0.45 \\
\hline T5 & 6.8 & 7.28 & 7.04 & 0.15 & 0.36 & 0.26 & 0.41 & 0.39 & 0.40 \\
\hline T6 & 7.0 & 7.24 & 7.12 & 0.15 & 0.27 & 0.21 & 0.47 & 0.42 & 0.45 \\
\hline T7 & 7.1 & 7.26 & 7.18 & 0.12 & 0.24 & 0.18 & 0.45 & 0.43 & 0.44 \\
\hline T8 & 7.1 & 7.24 & 7.17 & 0.14 & 0.29 & 0.22 & 0.47 & 0.48 & 0.48 \\
\hline T9 & 7.2 & 7.27 & 7.24 & 0.13 & 0.21 & 0.17 & 0.43 & 0.41 & 0.42 \\
\hline T10 & 6.9 & 7.24 & 7.07 & 0.14 & 0.15 & 0.15 & 0.48 & 0.44 & 0.46 \\
\hline T11 & 7.1 & 7.18 & 7.14 & 0.13 & 0.24 & 0.19 & 0.47 & 0.45 & 0.46 \\
\hline T12 & 7.1 & 7.14 & 7.12 & 0.14 & 0.18 & 0.16 & 0.48 & 0.52 & 0.50 \\
\hline T13 & 6.9 & 7.38 & 7.14 & 0.14 & 0.27 & 0.21 & 0.41 & 0.42 & 0.42 \\
\hline SEm \pm & 0.095 & 0.094 & 0.043 & 0.012 & 0.027 & 0.014 & 0.03 & 0.04 & 0.025 \\
\hline CD $(0.05)$ & NS & NS & NS & NS & NS & 0.041 & 0.08 & NS & 0.073 \\
\hline CV\% & 2.35 & 2.28 & 1.06 & 17.28 & 19.33 & 13.71 & 11.33 & 15.93 & 9.96 \\
\hline Initial value & 6.9 & 7.68 & 7.29 & 0.12 & 0.27 & 0.19 & 0.41 & 0.45 & 0.43 \\
\hline
\end{tabular}


Table.5 Effect of fertilizer levels on soil available macronutrients $(\mathrm{kg} / \mathrm{ha})$ of finger millet

\begin{tabular}{|l|c|c|c|c|c|c|c|c|c|}
\hline \multirow{2}{*}{ Treatment } & \multicolumn{3}{|c|}{ Available N (kg/ha) } & \multicolumn{3}{c|}{ Available P2O5 (kg/ha) } & \multicolumn{3}{c|}{ Available K2O (kg/ha) } \\
\cline { 2 - 10 } & $\mathbf{2 0 1 5}$ & $\mathbf{2 0 1 6}$ & Mean & $\mathbf{2 0 1 5}$ & $\mathbf{2 0 1 6}$ & Mean & $\mathbf{2 0 1 5}$ & $\mathbf{2 0 1 6}$ & Mean \\
\hline T1 & 185 & 125 & 155 & 79 & 56 & 68 & 254 & 255 & 255 \\
\hline T2 & 189 & 129 & 159 & 82 & 59 & 71 & 257 & 261 & 259 \\
\hline T3 & 173 & 121 & 147 & 80 & 47 & 64 & 241 & 228 & 235 \\
\hline T4 & 178 & 130 & 154 & 82 & 53 & 68 & 257 & 241 & 249 \\
\hline T5 & 194 & 163 & 179 & 78 & 66 & 72 & 266 & 252 & 259 \\
\hline T6 & 198 & 167 & 183 & 80 & 69 & 75 & 271 & 246 & 259 \\
\hline T7 & 224 & 180 & 202 & 69 & 85 & 77 & 290 & 270 & 280 \\
\hline T8 & 211 & 184 & 198 & 78 & 88 & 83 & 293 & 279 & 286 \\
\hline T9 & 203 & 167 & 185 & 84 & 70 & 77 & 266 & 246 & 256 \\
\hline T10 & 206 & 172 & 189 & 90 & 74 & 82 & 275 & 258 & 267 \\
\hline T11 & 224 & 184 & 204 & 88 & 82 & 85 & 283 & 285 & 284 \\
\hline T12 & 236 & 188 & 212 & 92 & 84 & 88 & 295 & 319 & 307 \\
\hline T13 & 182 & 125 & 154 & 73 & 44 & 59 & 206 & 184 & 195 \\
\hline SEm \pm & 11.16 & 11.40 & 8.169 & 8.30 & 4.49 & 4.87 & 18.05 & 16.72 & 13.23 \\
\hline CD $(0.05)$ & 32.56 & 33.27 & 23.84 & NS & 13.12 & 14.21 & 52.67 & 48.80 & 38.61 \\
\hline CV\% & 9.66 & 12.60 & 7.93 & 17.70 & 11.53 & 11.33 & 11.75 & 11.33 & 8.78 \\
\hline Initial value & 134 & 127 & 131 & 99 & 65 & 8.2 & 203 & 253 & 228 \\
\hline
\end{tabular}

Table.6 Effect of fertilizer levels on Soil available micronutrients $(\mathrm{kg} / \mathrm{ha})$ of finger millet

\begin{tabular}{|c|c|c|c|c|c|c|c|c|c|c|c|c|}
\hline \multirow[t]{2}{*}{ Treatment } & \multicolumn{3}{|c|}{ Available Zn (ppm) } & \multicolumn{3}{|c|}{ Available Fe (ppm) } & \multicolumn{3}{|c|}{ Available Mn (ppm) } & \multicolumn{3}{|c|}{ Available Cu (ppm) } \\
\hline & 2015 & 2016 & Mean & 2015 & 2016 & Mean & 2015 & 2016 & Mean & 2015 & 2016 & Mean \\
\hline $\mathrm{T} 1$ & 1.78 & 0.80 & 1.29 & 8.37 & 5.09 & 6.73 & 9.34 & 5.38 & 7.36 & 1.38 & 2.94 & 2.16 \\
\hline $\mathrm{T} 2$ & 1.92 & 0.91 & 1.42 & 8.10 & 5.54 & 6.82 & 9.65 & 6.17 & 7.91 & 1.51 & 1.92 & 1.72 \\
\hline T3 & 1.67 & 0.72 & 1.20 & 8.18 & 5.69 & 6.94 & 9.01 & 6.08 & 7.55 & 1.43 & 2.40 & 1.92 \\
\hline $\mathrm{T} 4$ & 1.73 & 1.00 & 1.37 & 8.22 & 5.85 & 7.04 & 8.33 & 7.11 & 7.72 & 1.45 & 1.97 & 1.71 \\
\hline T5 & 1.70 & 0.95 & 1.33 & 8.28 & 6.08 & 7.18 & 9.29 & 7.09 & 8.19 & 1.43 & 3.01 & 2.22 \\
\hline T6 & 1.85 & 0.99 & 1.42 & 8.45 & 5.71 & 7.08 & 9.48 & 7.25 & 8.37 & 1.49 & 3.24 & 2.37 \\
\hline T7 & 1.99 & 0.79 & 1.39 & 8.77 & 5.54 & 7.16 & 10.08 & 8.18 & 9.13 & 1.63 & 3.01 & 2.32 \\
\hline T8 & 2.11 & 0.86 & 1.49 & 8.48 & 6.91 & 7.70 & 10.48 & 8.71 & 9.60 & 1.65 & 3.66 & 2.66 \\
\hline T9 & 1.82 & 0.91 & 1.37 & 8.27 & 5.83 & 7.05 & 9.55 & 7.10 & 8.33 & 1.60 & 2.21 & 1.91 \\
\hline $\mathrm{T} 10$ & 1.94 & 0.94 & 1.44 & 8.64 & 6.89 & 7.77 & 9.68 & 7.26 & 8.47 & 1.62 & 2.54 & 2.08 \\
\hline $\mathrm{T} 11$ & 2.08 & 1.03 & 1.56 & 8.94 & 5.36 & 7.15 & 9.96 & 7.16 & 8.56 & 1.66 & 3.15 & 2.41 \\
\hline $\mathrm{T} 12$ & 2.34 & 1.09 & 1.72 & 8.85 & 6.20 & 7.53 & 10.21 & 7.40 & 8.81 & 1.78 & 3.39 & 2.59 \\
\hline T13 & 1.39 & 0.84 & 1.12 & 7.91 & 5.98 & 6.95 & 9.13 & 5.01 & 7.07 & 1.35 & 1.38 & 1.37 \\
\hline SEm \pm & 0.16 & 0.088 & 0.087 & 0.40 & 0.333 & 0.251 & 0.46 & 0.549 & 0.279 & 0.15 & 0.237 & 0.140 \\
\hline $\mathrm{CD}(0.05)$ & 0.47 & NS & 0.254 & NS & 0.97 & NS & 1.33 & 1.60 & 0.814 & NS & 0.69 & 0.409 \\
\hline $\mathrm{CV} \%$ & 14.79 & 16.77 & 10.83 & 8.31 & 9.78 & 6.08 & 8.25 & 13.75 & 5.87 & 17.40 & 15.39 & 11.51 \\
\hline $\begin{array}{l}\text { Initial } \\
\text { value }\end{array}$ & 1.64 & 0.78 & 1.21 & 7.86 & 5.17 & 6.515 & 9.18 & 6.54 & 7.86 & 1.23 & 3.03 & 2.13 \\
\hline
\end{tabular}


Table.7 Effect of fertilizer levels on plant macronutrient uptake $(\mathrm{kg} / \mathrm{ha})$ of finger millet

\begin{tabular}{|l|c|c|c|c|c|c|c|c|c|}
\hline \multirow{2}{*}{ Treatment } & \multicolumn{3}{|c|}{ N Uptake (kg/ha) } & \multicolumn{3}{c|}{ P Uptake (kg/ha) } & \multicolumn{3}{c|}{ K Uptake (kg/ha) } \\
\cline { 2 - 11 } & $\mathbf{2 0 1 5}$ & $\mathbf{2 0 1 6}$ & Mean & $\mathbf{2 0 1 5}$ & $\mathbf{2 0 1 6}$ & Mean & $\mathbf{2 0 1 5}$ & $\mathbf{2 0 1 6}$ & Mean \\
\hline T1 & 46.0 & 50.37 & 48.2 & 14.5 & 17.55 & 16.0 & 54.93 & 65.78 & 60.4 \\
\hline T2 & 54.1 & 58.38 & 56.2 & 17.3 & 20.91 & 19.1 & 64.58 & 72.40 & 68.5 \\
\hline T3 & 48.0 & 48.41 & 48.2 & 13.5 & 15.12 & 14.3 & 53.85 & 58.32 & 56.1 \\
\hline T4 & 51.6 & 55.76 & 53.7 & 14.8 & 17.73 & 16.3 & 58.36 & 71.00 & 64.7 \\
\hline T5 & 58.9 & 72.35 & 65.6 & 20.2 & 23.48 & 21.8 & 71.73 & 74.36 & 73.0 \\
\hline T6 & 61.8 & 80.76 & 71.3 & 22.2 & 25.88 & 24.0 & 77.52 & 79.89 & 78.7 \\
\hline T7 & 74.8 & 86.09 & 80.4 & 28.3 & 27.21 & 27.8 & 87.45 & 82.11 & 84.8 \\
\hline T8 & 77.7 & 96.01 & 86.9 & 30.4 & 30.08 & 30.2 & 91.46 & 93.65 & 92.6 \\
\hline T9 & 62.3 & 68.60 & 65.5 & 19.0 & 23.44 & 21.2 & 70.45 & 73.42 & 71.9 \\
\hline T10 & 65.9 & 77.28 & 71.6 & 20.1 & 26.74 & 23.4 & 74.61 & 82.31 & 78.5 \\
\hline T11 & 76.8 & 88.62 & 82.7 & 27.2 & 30.13 & 28.7 & 86.47 & 87.81 & 87.1 \\
\hline T12 & 80.9 & 98.39 & 89.6 & 29.6 & 35.47 & 32.5 & 89.53 & 96.51 & 93.0 \\
\hline T13 & 36.5 & 49.28 & 42.9 & 16.7 & 17.82 & 17.3 & 34.83 & 58.94 & 46.9 \\
\hline SEm \pm & 3.19 & 3.34 & 2.07 & 1.61 & 0.995 & 0.89 & 6.28 & 4.08 & 3.59 \\
\hline CD $(0.05)$ & 9.43 & 9.73 & 6.07 & 4.70 & 2.91 & 2.60 & 18.35 & 11.93 & 10.47 \\
\hline CV\% & 9.06 & 8.07 & 5.42 & 13.74 & 7.20 & 6.86 & 15.46 & 9.24 & 8.45 \\
\hline
\end{tabular}

Table.8 Effect of fertilizer levels on plant micronutrient uptake (gm/ha) of finger millet

\begin{tabular}{|l|c|c|c|c|c|c|}
\hline Treatment & \multicolumn{3}{|c|}{ Zn Uptake } & \multicolumn{3}{c|}{ Fe Uptake } \\
\hline & $\mathbf{2 0 1 5}$ & $\mathbf{2 0 1 6}$ & Mean & $\mathbf{2 0 1 5}$ & $\mathbf{2 0 1 6}$ & Mean \\
\hline T1 & 209.0 & 207.3 & 202.4 & 659 & 1024 & 1104.1 \\
\hline T2 & 238.9 & 251.2 & 251.5 & 814 & 1240 & 1375.5 \\
\hline T3 & 197.4 & 185.7 & 179.7 & 593 & 944 & 993.3 \\
\hline T4 & 209.7 & 210.7 & 203.3 & 663 & 999 & 1087.4 \\
\hline T5 & 235.6 & 237.1 & 242.5 & 804 & 1112 & 1300.0 \\
\hline T6 & 255.1 & 274.6 & 281.2 & 911 & 1258 & 1492.7 \\
\hline T7 & 276.3 & 320.7 & 313.4 & 1031 & 1309 & 1572.9 \\
\hline T8 & 295.0 & 351.2 & 343.4 & 1097 & 1409 & 1681.5 \\
\hline T9 & 277.5 & 275.7 & 301.6 & 964 & 1075 & 1460.1 \\
\hline T10 & 308.1 & 321.5 & 352.1 & 1025 & 1271 & 1619.3 \\
\hline T11 & 349.8 & 337.7 & 383.7 & 1176 & 1301 & 1697.2 \\
\hline T12 & 367.0 & 369.0 & 405.9 & 1282 & 1441 & 1828.8 \\
\hline T13 & 168.4 & 180.7 & 149.4 & 434 & 944 & 826.2 \\
\hline SEm \pm & 11.41 & 14.22 & 13.35 & 73.75 & 54 & 84.70 \\
\hline CD $(0.05)$ & 33.28 & 41.50 & 38.97 & 215.25 & 158 & 247.20 \\
\hline CV\% & 12.80 & 9.09 & 8.33 & 14.49 & 7.97 & 10.57 \\
\hline
\end{tabular}


The soil available Nitrogen, Phosphorus and Potassium were significantly influenced by various treatments in which fertilizers were applied based on different approaches and integrated nutrient supply (Table 5). The available Nitrogen in the soil after the harvest of the crop was found highest in the treatment T12 with $200 \%$ RDN $+100 \%$ RDP $+100 \%$ $\mathrm{RDK}+25 \% \mathrm{RDZn}+25 \% \mathrm{RDS}+25 \% \mathrm{RDB}$ integrated with FYM @ 5t ha ${ }^{-1}\left(212 \mathrm{~kg} \mathrm{ha}^{-1}\right)$ on par with T11 followed by $\mathrm{T} 7$ and $\mathrm{T} 8$ treatments in which fertilizers were applied based on STCR equation for a targeted yield of $40 \mathrm{q} \mathrm{ha}^{-1}$ and treatment $\mathrm{T} 10$.

The available phosphorus in the soil was found highest in the treatment T12 with $200 \%$ $\mathrm{RDN}+100 \% \mathrm{RDP}+100 \% \mathrm{RDK}+25 \%$ $\mathrm{RDZn}+25 \% \mathrm{RDS}+25 \% \mathrm{RDB}$ integrated with FYM @ 5t ha ${ }^{-1}\left(88 \mathrm{~kg} \mathrm{ha}^{-1}\right)$ on par with the treatments T11, T6, T7, T8, T9, T10. The soil available $\mathrm{K}_{2} \mathrm{O}$ was found highest in the treatment T12 (307 $\mathrm{kg} \mathrm{ha}^{-1}$ ) which was on par with the treatments T7, T8 and T11. Similar results were reported by Kumara et al., (2014), Saravanane et al., (2011) and Shivakumar et al., (2011).

Among the soil available micronutrients, available $\mathrm{Zn}, \mathrm{Mn}$ and $\mathrm{Cu}$ were significantly influenced by various treatments. The soil available $\mathrm{Zn}$ was found highest in the treatment T12 with $200 \% \mathrm{RDN}+100 \%$ RDP $+100 \% \mathrm{RDK}+25 \% \mathrm{RDZn}+25 \% \mathrm{RDS}+$ $25 \%$ RDB integrated with FYM @ 5t ha ${ }^{-1}$ (1.72 ppm) which was on par with treatment T11 and T8 in which the fertilizers were applied based on STCR equation for target yield of 40q/ha and integrated with FYM @ 5t/ha. Soil available $\mathrm{Mn}$ and $\mathrm{Cu}$ was found highest in the treatment T8 $(9.60 \mathrm{ppm}$ and $2.66 \mathrm{ppm})$ in which fertilizers were applied based on STCR equation for a targeted yield of $40 \mathrm{q} \mathrm{ha}^{-1}$ integrated with FYM @ 5t ha ${ }^{-1}$ which is on par with T11, T12, T6 and T7. The soil available Fe was not significantly influenced due to the application of nutrients on the basis of different approaches (Table 6).

The uptake of macronutrients by finger millet plant (N, P and $\mathrm{K}$ ) was significantly influenced by various treatments. The plant uptake of $\mathrm{N}$ and $\mathrm{P}$ was found highest in the treatment T12 with $200 \% \mathrm{RDN}+100 \% \mathrm{RDP}$ $+100 \% \mathrm{RDK}+25 \% \mathrm{RDZn}+25 \% \mathrm{RDS}+$ 25\% RDB integrated with FYM @ 5t ha ${ }^{-1}$ (89.6 kg ha ${ }^{-1}$ and $32.5 \mathrm{~kg} \mathrm{ha}^{-1}$ ) which was on par with the treatment T8. The uptake of potassium was found highest in the treatment T12 with $200 \%$ RDN $+100 \%$ RDP $+100 \%$ $\mathrm{RDK}+25 \% \mathrm{RDZn}+25 \% \mathrm{RDS}+25 \% \mathrm{RDB}$ integrated with FYM @ 5t ha ${ }^{-1}(93.0$ kg/ha) which was on par with the treatments T11, T8 and $\mathrm{T} 7$ in which the fertilizers are applied based on STCR equation for a targeted yield of $40 \mathrm{q} \mathrm{ha} \mathrm{h}^{-1}$ integrated with FYM. The uptake of $\mathrm{Zn}$ and $\mathrm{Fe}$ was found highest in the treatment T12 (405.9 $\mathrm{gm} \mathrm{ha}^{-1}$ and $1828.8 \mathrm{gm}$ $\mathrm{ha}^{-1}$ ) which is on par with the treatments T8 and T11 (Tables 7 and 8). The Benefit cost ratio (Table 3) was found highest in the treatment T7 (3.30) in which the fertilizers were applied based on STCR equation for a targeted yield of $40 \mathrm{q} \mathrm{ha}{ }^{-1}$ which was very closely followed by treatment T11 (3.27).

In conclusion, these values suggest that soils with low fertility status of available Nitrogen less than $140 \mathrm{~kg} / \mathrm{ha}$, increase in the $\mathrm{N}$ dose upto $200 \%$ along with micronutrients can give yields on par with the nutrient supply using STCR approach. Hence it can be concluded that the targeted yield in finger millet could be achieved with integrated nutrient supply using STCR approach.

The higher productivity of finger millet may be attributed to improved root growth, nutrient uptake, simulation of many different enzymes related to photosynthesis, improved soil properties and increased nutrient use efficiency of applied nutrients. 


\section{References}

Anil Kumar, B.H., Sharanappa, K. T., Krishne Gowda., and Sudhir, K. 2003. Growth, yield and nutrient uptake as influenced by integrated nutrient management in dry land finger millet. Mysore Journal of Agricultural Sciences. 37(1): $24-28$.

Apoorva, K.B., Prakash, S.S., Rajesh, N.L., and Nandini, B. (2010). STCR Approach for Optimising Integrated Plant Nutrient Supply on Growth, Yield and Economics of Finger Millet (Eleusine coracana (L.) Garten.). European Journal of Biological Sciences. 4(1): 19-27.

Chen, X.P., Cui, Z.L., Vitousek, P.M., Cassman, K.G., Matson, P.A., Bai, J.S., Meng, Q.F., Hou, P., Yue, S.C., Romheld, V., and Zhang, F.S. (2011). Integrated soil-crop system management for food security. Proceedings of National Academy Sciences, USA. 108, 6399-6404.

Devi, P.B., Vijayabharathi, R., Satyabhama, S., Malledhi, N.G., and Priyadarshini, V.B. 2011. Health Benefits of Finger millet (Eleusinecoracana L.) Polyphenols and Dietary Fiber. A Review, Journal of Food Science Technology. DOI: 10.1007/S13197011-0584-9.

Drinkwater, L.E. and Snapp, S.S. (2007). Nutrients in Agroecosystems: Rethinking the management paradigm. Advances in Agronomy. 92, 163-186.

Duryodhana, D., Krishnegowda, K. T., Kadalli, G. G., and Ashok, E. G. 2004. Uptake pattern of major nutrients at different growth stages by dry land finger millet (Eleusine coracana (L.) Gaertn.) From different sources and levels of nutrients. Mysore Journal of Agricultural Sciencies. 38(4): 487 495.
Hemalatha, S., and Chellamuthu, S. 2011. Effect of long term fertilization on phosphorous fractions under finger millet-maize cropping sequence. Madras Agricultural Journal. 98, 344346.

Hemalatha, S., and Chellamuthu, S. 2013. Impacts of long term fertilization on soil nutritional quality under finger millet: Maize cropping sequence. Journal of Environmental Research Developments. 7, 1571-1576.

Kumara, O., Naik, T.B. and Palaiah, P. 2007. Effect of weed management practices and fertility levels on growth and yield parameters in finger millet. Karnataka Journal of Agricultural Sciences. 20, 230-233.

Kumara, O., Naik, T.B., and Ananadakumar, B.M. 2014. Effect weed management practices and fertility levels on soil health in finger millet-groundnut cropping system. International Journal of Agricultural Sciences. 10, 351-355.

Malakouti, M. J. 2008. "Zinc is a Neglected element in the life cycle of plants: A review". Middle Eastern and Russian Journal of Plant Science and Biotechnology. Vol 1, No.1, pp. 1-12.

Pingali, P., and Pandey, S. 2005. "Meeting World Maize Needs: Technological Opportunities and Priorities for the Public Sector", CIMMYT World Maize Facts and Trends. Vol. 93, No.2-3, pp. 252-263.

Sankar, G.R.M., Sharma, K.L., Dhanapal, G.N., Shankar, M.A., Mishra, P.K., Venkateswarlu, B., and Grace, J.K. 2011. Influence of soil and fertilizer nutrients on sustainability of rainfed finger millet yield and soil fertility in semi-arid Alfisols. Communication of Soil Science and Plant Analysis. 42, 1462-1483.

Saravanane, P., Nanjappa, H.V., Ramachandrappa, B.K., and Soumya, T. 
2011. Effect of residual fertility of preceding potato crop on yield and nutrient uptake of finger millet. Karnataka Journal Agricultural Sciences. 24, 234-236.

Shivakumar, B.C., Girish, A.C., Gowda, B., Kumar, G.C.V., Mallikarjuna, A.P., and Thimmegowda, M.N. 2011. Influence of pongamia, mahua and neem cakes on finger millet productivity and soil fertility. Journal of Applied Natural Sciences. 3, 274-276.

Singh, D.K., Pandey, A.K., Pandey, U.B., and Bhonde, S.R. 2002. "Effect of Farmyard Manure combined with Foliar Application of NPK Mixture and Micronutrients on Growth, Yield and Quality of Onion", Newsletter-National Horticulture Research Development Foundation. Vol.21-22, No. 1, pp.1-7.

Singh, K.N., Raju, N.S., Subba, A.R., Abhishek, R., Sanjay, S., Samanta, R.K., and Maji. 2005. Prescribing optimum dose of nutrients for targeted yield through soil fertility map in
Andhra Pradesh (AP). Journal of Indian Society Agricultural Statistics. 59(2):131-140.

Srinivasarao, C., Venkateswarlu, B., Singh, A.K., Vittal, K.P.R., Kundu, S., Chary, G.R., Gajanan, G.N., and Ramachandrappa, B.K. 2012. Critical carbon inputs to maintain soil organic carbon stocks under long-term fingermillet (Eleusine coracana [L.] Gaertn.) Cropping on Alfisols in semiarid tropical India. Journal of Plant Nutrition and Soil Science. 175, 681-688.

Tandon, H.L.S. 1997. "Experience with Balanced Fertilization in India". Better crops. Vol. 11, No.1, PP.20-21.

Wu, W., and Ma, B. 2015. Integrated nutrient management (INM) for sustaining crop productivity and reducing environmental impact: A review. Science and Total Environment. 512513, 415-427.

Treen Hein. 2005. Forging ahead with finger millet. New Agriculturist Developments, pp.1-2.

\section{How to cite this article:}

Sandhya Rani, Y., U. Triveni, T.S.S.K. Patro, M. Divya and Anuradha, N. 2017. Revisiting of Fertilizer Doses in Finger Millet [Eleusine coracana (L.) Garten.] Through Targeted Yield and Soil Test Crop Response (STCR) Approach. Int.J.Curr.Microbiol.App.Sci. 6(7): 2211-2221. doi: https://doi.org/10.20546/ijcmas.2017.607.321 